\title{
Propuesta para el diseño de un sistema de indicadores integrado para la coyuntura turística de Canarias
}

\author{
Yasmina TORRES LORENZO \\ Raúl MARTÍN HERNÁNDEZ \\ Máster en Dirección y Planificación del Turismo \\ Universidad de La Laguna \\ yasminatorres88@gmail.com
}

Recibido: 10-01-2013

Aceptado: 21-01-2013

\section{RESUMEN}

Vivimos en un contexto en el que la información, más que suponer una carencia, puede llegar a representar un exceso, y, esto, a la hora de tomar decisiones en un sector como el turístico, tan importante para la economía de Canarias, puede dificultar enormemente esta labor.

Dar solución a este problema es, en esencia, la razón de ser de este proyecto, que se presenta como una propuesta para el diseño de un sistema de indicadores con el que explicar la situación coyuntural de Canarias (aunque podría un modelo perfectamente extrapolable a otras regiones).

Partiendo de un trabajo con tres niveles de información (indicadores base o elementales, indicadores clave y un indicador sintético), se propone un modelo en formato web, de visualización rápida y sencilla, que aporte información valiosa para explicar la situación coyuntural turística de Canarias, y apoyar así la toma de decisiones pública y privada.

Palabras clave: Turismo, coyuntura, Canarias, indicadores base, indicadores clave, indicador sintético, sistema de indicadores 


\title{
Proposal for designing an indicator system to know the Canarian short-term touristic perspective
}

\begin{abstract}
In the current context of excess of information, to make decisions is not easy, and if we are talking about a sector like Tourism, one of the most important sources of revenue for the Canary Islands, this is even harder.

Solving this problem is the reason why this project appears, with the intention of creating an indicator system with whom to explain the short-term touristic perspective, in this case for the Canary Islands (although it can be applied to other regions).

For this project, we have worked with three levels of information (basic, key and synthetic indicators), trying to establish a useful tool in web format, very simple and with a quick view, for helping us to understand the current status of Tourism. The main objective of this work is to help to make decisions in the public and private sector.
\end{abstract}

Keywords: Tourism, tourism situation, tourism scenario, Canary Islands, basic indicators, key indicators, synthetic indicators, indicator system

Sumario: 1. Introducción 2. Objetivos y relevancia del proyecto 3. Antecedentes 4. Propuesta metodológica 5. Conclusiones

\section{INTRODUCCIÓN}

El turismo constituye en la actualidad una de las actividades más relevantes en la mayoría de las economías modernas, ubicándose entre las exportaciones más importantes a nivel mundial, lo que se traduce en una creciente necesidad de disponer de información que nos permita conocer las condiciones actuales y futuras de la actividad turística. Y es que aumenta, no solo la importancia cuantitativa del turismo, sino también la cualitativa, y esto pone de manifiesto el requerimiento de herramientas que permitan un análisis más profundo de la situación del sector que nos legitime para dar respuesta a múltiples preguntas. Partiendo de esta idea, encontramos que, unido a la peculiar situación económica vivida desde el comienzo de la crisis, y a los continuos cambios que se están produciendo en el sector, en gran parte, explicados por el auge de las nuevas tecnologías, cobran vital importancia la investigación y, especialmente, el análisis coyuntural, que trata de conocer, con la mayor brevedad posible, la situación y el ritmo de crecimiento de la actividad turística y los factores más probables de su evolución.

A partir de este contexto donde la importancia del análisis coyuntural en turismo es cada vez un aspecto más vital, tiene sentido comenzar a tratar de manera más específica el supuesto de Canarias, caso que establece los cimientos sobre los que se ha construido el presente proyecto, sin perjuicio de poder constituir igualmente un modelo extrapolable a otras Comunidades Autónomas. 
Al hablar sobre el sector turístico en Canarias, nos encontramos ante una actividad de vital importancia para la economía de las islas, que desempeña un papel fundamental en la generación de renta y empleo, y con unas cifras de PIB en torno al $29,5 \%$ sobre el conjunto de la economía regional (2011) ${ }^{1}$. Asimismo, las cifras de turistas recibidos son muy significativas y tan solo superadas, a nivel nacional, por Cataluña y Baleares. Fueron 11.624 .062 los turistas que eligieron Canarias como destino durante el año 2012 (un 2,39\% menos que en el año 2011), representando éstos un $17,58 \%$ del total de turistas entrados a España. En cuanto a la distribución de los mismos en función de sus lugares de residencia, destaca el predominio reinante de los extranjeros sobre los residentes en España, con un $87,13 \%$ del total frente al $12,87 \%$ restante representado por éstos últimos. Atendiendo a la llegada de visitantes por islas, Tenerife se situó en 2012 como la principal receptora de turistas, con casi cuatro millones y medio de visitantes, seguida de Gran Canaria, con algo más de tres millones, Lanzarote, que superó ligeramente los dos millones y Fuerteventura, que casi rozó esta cifra. Muy de lejos, se encontraron las islas de menor tamaño: La Palma, La Gomera y El Hierro ${ }^{2}$.

En contraposición a la disminución observada en el número de turistas en 2012 con respecto al año 2011, llama la atención cómo el gasto total presenta una tendencia creciente a lo largo de los últimos años, con incrementos del $14,90 \%$ en 2011 y $3,61 \%$ en 2012, en relación a sus respectivos años anteriores. Sin embargo, al detenernos en el desglose del mismo teniendo en cuenta dónde fue efectuado, se puede observar cómo el gasto en destino ha caído considerablemente entre 2011 y 2012 (con una tasa de variación anual del $-3,31 \%$ ), al mismo tiempo que ha aumentado el gasto realizado en origen (con un crecimiento del $6,82 \%)^{3}$.

Para hacer frente al gran número de turistas que recibe anualmente, Canarias cuenta con una importante infraestructura alojativa que se resume en 2.466 establecimientos, englobándose hoteles, apartamentos, alojamiento de tipo rural y campings, con una capacidad total de 442.742 plazas. $^{4}$

Después de observar tan importantes cifras, no queda duda alguna de lo esencial que es la actividad turística en la Comunidad Autónoma de Canarias, constituyendo un destino turístico ejemplar a nivel internacional, lo que acentúa la necesidad de contar con información estadística de carácter coyuntural, relevante y adecuadamente estructurada, que legitime la toma de decisiones en la materia.

\footnotetext{
${ }^{1}$ Según Exceltur y Gobierno de Canarias (2012), IMPACTUR Canarias 2011

2 Según datos de FRONTUR- Canarias., Estadística de Movimientos Turísticos en Fronteras de Canarias, ISTAC

${ }^{3}$ Según datos de la Encuesta Sobre Gasto Turístico, ISTAC

4 A partir de los datos de la Encuesta de Alojamiento Turístico en Establecimientos Hoteleros y la Encuesta de Alojamiento Turístico en Establecimientos Extrahoteleros, ISTAC, y Encuesta de ocupación en campings y Encuesta de ocupación en alojamientos de turismo rural, INE, 2012. Se trata de plazas totales, es decir, no solo las autorizadas
} 


\section{OBJETIVOS Y RELEVANCIA DEL PROYECTO}

El objetivo fundamental del presente proyecto es realizar una propuesta de los pilares sobre los que construir un sistema de indicadores que contenga información sobre la situación coyuntural del sector turístico en Canarias, con las siguientes características:

- De visualización rápida y sencilla que permita la construcción de un widget html, incrustable en otras páginas web de asociaciones empresariales, ayuntamientos, empresas, universidades, grupos de investigación, etc.

- Donde se puedan consultar los indicadores más importantes para determinar si la marcha del sector está siendo positiva o no, sin la necesidad de realizar largas búsquedas de información, recorriendo cada uno de los enlaces de la web del ISTAC correspondiente a cada operación estadística

- Valiosa para la toma de decisiones por parte de empresarios y Administraciones públicas

- De actualización instantánea, de forma que siempre se encuentre publicado el último dato disponible en la web del ISTAC, sin perjuicio de lo cual también podrá disponerse de cifras ya publicadas con anterioridad

- Con información de frecuencia temporal equivalente a la mensual, salvo en determinados casos en los que el mínimo disponible será el trimestre

- Que ofrezca datos a un nivel de desagregación territorial importante, al menos a escala municipal, entendiendo que cada municipio turístico de Canarias tiene sus características propias

- Personalizable, con la posibilidad de que el usuario pueda elegir algún indicador no contemplado en la selección predeterminada, pero que pueda resultar de su interés.

Dada la importancia del sector turístico en las Islas Canarias, cada vez se hace más indispensable la existencia de una herramienta que sirva de apoyo para la toma de decisiones tanto en el ámbito público como en el privado. De ahí la relevancia de este proyecto, que pretende sentar las bases para la construcción de un sistema de indicadores sobre la situación coyuntural del sector turístico en Canarias, que posibilite que los agentes económicos, públicos y privados, tengan conocimiento de en qué contexto se mueven, y puedan, incluso, realizar predicciones de cara al futuro, teniendo en cuenta el comportamiento que ha mostrado el sector en los últimos años.

Nos encontraríamos ante un instrumento clave en la toma de decisiones que pretende aportar información muy valiosa de cara a orientar las estrategias y líneas de actuación más adecuadas en materia turística al máximo nivel de desagregación territorial posible, a escala municipal, asumiéndose que, dentro de cada isla, los municipios turísticos muestran realidades muy heterogéneas. 
Pero, sin duda, la mayor relevancia del presente proyecto va unida intrínsecamente a su utilidad. En este punto conviene hacer referencia a la figura que representa el Instituto Canario de Estadística (ISTAC), órgano principal dentro del sistema de estadística de la Comunidad Autónoma de Canarias y, además, centro oficial de investigación autonómico. Entre sus funciones principales, habría que destacar dos fundamentalmente: por un lado, la de aportar información propia de carácter estadístico a un nivel de desagregación que no ofrecen otros organismos nacionales, bajo el cumplimiento de los principios establecidos en el Código de Buenas Prácticas de las Estadísticas Europeas (2005) publicado por EUROSTAT, $\mathrm{y}$, por otro, la de coordinar, gestionar y promover la actividad estadística pública.

En lo referente al sector turístico, no queda duda de que el ISTAC ha conseguido con creces su cometido de ofrecer operaciones estadísticas muy completas, sirviendo, en gran medida, como ejemplo para otras Comunidades Autónomas, con publicaciones llevadas a cabo, en muchos casos, con periodicidad mensual, y a un nivel de desagregación geográfica que incluso supera las exigencias europeas en la materia. Sin embargo, si nos ponemos en la piel de un gestor turístico, ya sea público o privado, a la hora de tomar una decisión y utilizando como respaldo la información publicada por el ISTAC, probablemente nos encontraríamos con que el volumen de datos es demasiado elevado y la ubicación de éstos se encuentra contenida en diferentes encuestas, lo que puede aportar ruido y dificultar la localización de la información más relevante y adecuada para el cometido que se pretende cumplir. De ahí surge la razón de ser del presente proyecto, que se propone como el punto de partida para la construcción de un sistema indicadores de coyuntura turística en el que se pueda visualizar de manera rápida y sencilla, los indicadores más relevantes para tener un conocimiento general sobre la marcha del sector, y que sirva de apoyo en la toma de decisiones en el ámbito público y privado.

\section{ANTECEDENTES}

Las publicaciones objeto de consulta para la elaboración de este proyecto, han sido revisadas en busca de información de varios tipos: referente a la metodología para la construcción de un sistema de indicadores, acerca del diseño del mismo y su posible visualización, y relativa a los pasos a seguir para la elaboración de un indicador sintético, que, si bien no ha sido desarrollado finalmente en el presente proyecto, sí que puede desprenderse una aproximación metodológica que puede constituir el punto de partida para futuras investigaciones.

\section{Publicaciones revisadas para la construcción del sistema}

En primer lugar, ha sido esencial conocer cuáles son las exigencias legislativas en el marco de las estadísticas del sector turístico a nivel europeo a través de la 
revisión del Reglamento (UE) No 692/2011 del Parlamento Europeo y del Consejo de 6 de julio de 2011, relativo a las estadísticas europeas sobre el turismo y por el que se deroga la Directiva 95/57/CE del Consejo. Se trata de un documento de gran importancia ya que constituye la base a partir de la cual ha de desarrollarse cualquier sistema de indicadores turísticos, estableciendo los requisitos exigibles en el ámbito europeo en términos de temporalidad y disponibilidad geográfica.

En términos de temporalidad, hay que destacar la consideración número 3, previa al articulado del citado reglamento, donde se refleja lo siguiente: "es preciso disponer de datos mensuales a fin de medir las repercusiones estacionales de la demanda sobre la capacidad del alojamiento turístico y ayudar así a las autoridades públicas y a los operadores económicos a preparar estrategias y líneas de actuación más adecuadas para mejorar el reparto de las actividades turísticas y las vacaciones a lo largo del año". Igualmente, en el apartado de anexos del Reglamento $\mathrm{N}^{\circ}$ 692/2011 del 6 de julio de 2011, también se hace referencia al mínimo temporal exigible para una serie de indicadores concretos, tanto desde el punto de vista de la oferta como del de la demanda, relacionados con el turismo interior. Estos límites temporales son de carácter mensual, anual o trienal, dependiendo del indicador. Teniendo en cuenta estas exigencias, en el presente proyecto se va a trabajar con datos de carácter mensual, en la mayor parte de los casos, y, en su defecto, con datos trimestrales, dada la disponibilidad de los datos de la Encuesta sobre Gasto Turístico, la de Expectativas Hoteleras, la de Empleo Registrado y la de Empresas Inscritas en la Seguridad Social.

En lo concerniente a la territorialidad, en la citada legislación se habla de ámbito nacional, para algunos indicadores, y de nivel regional NUTS 2, para otros. A modo aclaratorio, se han realizado tres tablas con la Nomenclatura de las Unidades Territoriales Estadísticas (NUTS, del francés Nomenclature d'unités territoriales statistiques) utilizadas por la Unión Europea con fines estadísticos para el caso de España.

En este sentido, cabría concluir que, a pesar de que el reglamento exige como mínimo la existencia de información a nivel autonómico, en el presente proyecto se van a utilizar los datos con los que trabaja el ISTAC, que entrarían en el grupo NUTS 3, por aportarse información por islas, llegando a profundizarse a un nivel aún más desagregado en el caso de los principales municipios turísticos de Canarias.

Asimismo, se ha utilizado como referencia otro documento llevado a cabo por el Instituto de Estadística y Cartografía de Andalucía: la metodología empleada para la elaboración de los llamados "Indicadores Estadísticos de Andalucía". Esta actividad, que forma parte del Plan Estadístico de Andalucía 2007- 2010 (que posteriormente fue extendido hasta el 31 de diciembre del 2012), "trata de mostrar un reflejo de la realidad andaluza, recopilando información acerca de todas aquellas variables relevantes referidas a Andalucía, y que permitan analizar la coyuntura regional y su comparación a nivel nacional, además de permitir en 
muchos casos una desagregación provincial". Para ello, se utiliza una amplia batería de indicadores sociales, demográficos, medioambientales y económicos, incluyéndose, en este último caso, la información acerca del sector turístico. En esta subárea se clasificarían una serie de actividades enfocadas a conocer la evolución del sector turístico, el comportamiento de la demanda turística y de la estructura y características básicas de los establecimientos turísticos, además de actuaciones públicas en el ámbito del turismo, planificación territorial, promoción e incentivos a la inversión.

\section{Diseño y visualización}

Una de las decisiones más complejas a tomar ha sido cómo mostrar la información recopilada por el sistema, de forma que los datos se mostraran de manera clara y sencilla, pero también dinámica, y con un componente cualitativo que traspasara las fronteras de las simples cifras llegando a aportar conocimiento de cuál es la marcha del sector. Esto es, no mostrar solamente el dato en sí, sino también algún indicativo de si la situación es favorable o desfavorable.

De obligada revisión han sido los informes sectoriales publicados de manera trimestral por la Cámara de Comercio de Santa Cruz de Tenerife en los que se presenta un completo listado de indicadores con datos reales y tasas de variación interanuales, tanto para Canarias como para España, a fin de poder realizar comparaciones con respecto a la media nacional. No obstante, esta publicación cuenta con dos limitaciones fundamentales que pretenden minimizarse en el presente proyecto, y son, por un lado, el contar con información trimestral y no mensual, $y$, por otro, el que dicha información no sea actualizada de manera permanente, lo que obliga a esperar hasta la siguiente publicación de la Cámara de Comercio. En un sector como el turístico, el poder obtener información sin apenas retardo temporal es un elemento enormemente valioso de cara a poder tomar decisiones a corto plazo, lo que enriquece, sin duda, el sistema de indicadores integrado propuesto.

Especial atención merece también la publicación Turismo en cifras, realizada por el Instituto de Estudios Turísticos, con un formato moderno y atractivo que facilita la comprensión de la información por parte de los usuarios.

Otro de los documentos revisados ha sido la Norma UNE 66175:2003. Guía para la implantación de sistemas indicadores, publicado por AENOR Ediciones, que muestra las experiencias de implantación de sistemas de indicadores en diversas empresas españolas, algunas de ellas con formas de visualización muy interesantes.

Igualmente, se han visitado algunas páginas web para obtener la documentación más enriquecedora posible, llegando hasta un foro en la red social profesional Linkedin, que considero que merece especial atención. El hilo del foro se titula Destination Management Organisations, y en él quedan de manifiesto los problemas, por parte de empresarios del sector turístico a nivel internacional, para 
acceder y, en muchos casos, interpretar las estadísticas de las que disponen, lo que, por consiguiente, les determina a la hora de tomar decisiones. Este interesante hallazgo, me llevó a plantearme en un primer momento si éste podía constituir un problema extrapolable a los gestores turísticos de Canarias, y esta hipótesis se vio confirmada, posteriormente, por don Juan Pablo González, gerente de ASHOTEL, en un encuentro que tuvo lugar el pasado mes de abril en las instalaciones del ISTAC (Santa Cruz de Tenerife).

\section{Publicaciones consultadas para la elaboración de un indicador sintético}

La presente propuesta para la construcción de un sistema de indicadores de coyuntura turística en Canarias parte de la idea de establecer tres niveles de información, donde se combinen indicadores base, indicadores clave y, por último, un índice sintético. Sin embargo, y como se explicará en apartados posteriores, este último nivel no llegará a completarse, por problemas metodológicos, pero sentará la base a partir de la cual seguir trabajando en futuros proyectos. Para determinar la metodología más adecuada para la elaboración del indicador sintético, se han revisado previamente algunos documentos, que se expondrán a continuación.

Por un lado, cabe señalar como referencia el documento de Anexos Metodológicos, publicado por Exceltur en Perspectivas Turísticas de Exceltur (2003). En este documento de diez páginas se recoge la metodología empleada por Exceltur para la realización de la Encuesta de Clima Turístico, la construcción del Indicador Sintético de Turismo en España (ISTE) y los llamados Modelos de Previsión, además de un anexo con definiciones. Nos hemos centrado, en concreto, en el apartado destinado a explicar la metodología llevada a cabo para la construcción del Indicador Sintético de Turismo en España (ISTE). Esta parte del documento comienza con un epígrafe destinado a la introducción en la materia, seguido de otro de explicación del proceso para la elaboración del indicador, para acabar con un último epígrafe de consideraciones finales.

Por otro lado, habría que hacer referencia a otro documento consultado para la elaboración del presente proyecto: las notas metodológicas del trabajo Indicadores de Actividad del Sector Servicios (IASS), tanto el referente a la base 2005 como el de base 2010, ambos elaborados por el Instituto Nacional de Estadística (INE) y publicados en abril de 2011 y marzo de 2013, respectivamente. Dichos trabajos recogen la metodología de la operación estadística de los Indicadores de Actividad del Sector Servicios, que tienen por objeto la medición a corto plazo de la evolución de la actividad de empresas no financieras operantes en el sector terciario en España a través de los indicadores cifra de negocios y personal ocupado. Vinculado a este documento, nos encontraríamos con otro, también revisado para la elaboración del presente proyecto, como ha sido el denominado Indicadores de Actividad del Sector Servicios, Metodología base 2010, elaborado por el Instituto de Estadística y Cartografía de Andalucía (IECA). En este caso, el IECA, partiendo de la información aportada por el indicador IASS del INE, ofrece 
datos más desagregados para la Comunidad Autónoma de Andalucía que los ya publicados a nivel nacional, adaptándose así a las necesidades de información concretas del sector servicios andaluz.

\section{PROPUESTA METODOLÓGICA}

El sistema de indicadores propuesto estaría compuesto por un conjunto de indicadores elementales, otros más relevantes, denominados clave y, por último, como proyecto futuro, uno sintético, fruto de la integración de tres de los anteriores.

La selección de cada uno de estos grupos de indicadores coincide con cada una de las fases de este proceso de construcción del sistema, y, si quisiéramos representarlo en una pirámide, cada grupo de indicadores se ubicaría en cada uno de los estamentos de la misma. Esto quiere decir que será necesario haber completado la base de esta pirámide para poder ascender hacia el siguiente nivel, y así sucesivamente a lo largo de la misma.

\section{Indicadores elementales o simples}

El primer paso para la construcción del sistema de indicadores propuesto ha consistido en la localización y selección de todos los indicadores de coyuntura turística que pudieran ser de interés, resultado de un importante proceso de consultas a diversas fuentes de ámbito regional, autonómico, nacional e internacional. Como parte de este proceso, se realizó un inventario con estos indicadores mediante el cual se obtuvieron metadatos acerca del estado de las estadísticas oficiales sobre coyuntura turística. Asimismo, se procedió a mostrar una información más detallada para cada uno de estos indicadores, definiéndose así su dimensión, el mínimo geográfico al que es posible acceder, su temporalidad y su unidad de medida. En el caso de la dimensión, se ha definido una clasificación genérica, atendiendo a la naturaleza de la mayoría de los indicadores, que podrían resumirse en: capacidad, ocupación, rentabilidad, confianza, empleo, afluencia, gasto y precios. Los indicadores que no encajan en ninguna de estas dimensiones han sido incluidos en un grupo llamado Otros.

Para la correcta interpretación de los indicadores, es necesario hacer una pequeña puntualización, y es que al hablar de alojamientos, solo se han tenido en cuenta los de tipo hotelero y extrahotelero (apartamentos turísticos) por considerarse los más relevantes. Es cierto que el segmento de turismo rural cada vez está cobrando más importancia, sobre todo en las islas de menor tamaño, pero es una evidencia que Canarias ha sido tradicionalmente y sigue siendo en la actualidad un destino eminentemente de Sol y Playa. Si observamos cuáles fueron los principales aspectos de elección de Canarias confesados por los turistas el pasado año 2012, tenemos que la motivación de mayor peso fue el clima o sol, con 
más de un $83 \%$ del total, seguida del relax $(37,87 \%)$ y las playas $(31,03 \%)$. Además, la información estadística que se recoge en la actualidad para este tipo de turismo es realmente escasa, si la comparamos con la vinculada a hoteles y apartamentos.

\section{Indicadores clave}

Una vez expuestos los indicadores elementales a tener en cuenta, el siguiente paso ha sido llevar a cabo una selección inicial de los indicadores más relevantes, de la que se obtuvo un total de 15. La recopilación de los indicadores más relevantes se ha realizado, en un primer lugar, en base a un criterio propio al que, posteriormente, se le añadiría otro de carácter más objetivo, que ha sido el nivel de uso de los indicadores (información con la que cuenta el propio ISTAC en función del número de búsquedas realizadas por los usuarios dentro de la página web). Con posterioridad, ambos criterios han sido validados por la opinión de expertos del sector. En este sentido se agradece una vez más la inestimable aportación, por un lado, del gerente de ASHOTEL (Asociación Hotelera y Extrahotelera de Tenerife, La Palma, La Gomera y El Hierro), don Juan Pablo González, que nos ha permitido tener un contacto mucho más realista con el sector desde el punto de vista del hotelero y extrahotelero, y por otro, de los Patronatos de Turismo de las islas, que nos aportaron su visión desde el enfoque del sector público.

A continuación se mostrará un listado con los indicadores clave seleccionados:

1- Llegadas de turistas que han elegido ese destino como el principal.

2- Llegadas de turistas que han elegido ese destino como el secundario

3- Excursionistas y pasajeros de cruceros

4- Plazas totales ofertadas (hoteleras y extrahoteleras)

5- Pernoctaciones hoteleras y extrahoteleras

6- Índice censal de ocupación hotelera y extrahotelera por plaza

7- Tarifa media diaria (ADR)

8- Ingresos por habitación disponible (RevPAR)

9- Ingresos totales

10- Índice de Confianza Hotelera

11- Total de empleos registrados

12- Paro registrado

13- Empresas inscritas en la seguridad social

14- Gasto turístico total (macromagnitud)

15- Gasto medio por turista y día 


\section{Indicador sintético: planteamiento base sobre el que trabajar en futuros proyectos}

En una idea inicial, se planteó la posibilidad de crear un indicador que sintetizara el gasto realizado por los turistas en destino y los ingresos totales generados por los mismos, mediante el producto del ADR correspondiente para cada tipo de establecimiento por las habitaciones ocupadas. El objetivo planteado inicialmente era obtener un indicador sintético que permitiera realizar el seguimiento de la actividad turística en Canarias, con: capacidad sintética, pudiendo reflejar el impacto de todas las actividades características del turismo; con la posibilidad de desagregación por islas y por mercados, a través de subindicadores; y con capacidad de determinar el impacto de cada uno de los subindicadores en el indicador total. En lo referente al gasto turístico, los datos serían extraídos de la Encuesta sobre Gasto Turístico de la web del ISTAC, utilizando, para ello, los valores absolutos correspondientes a cada una de las islas desde el primer trimestre del 2009 hasta el primero de 2013. Una vez se hubiera contado con dicha información, se procedería al cálculo del índice. El periodo establecido como base sería el primer trimestre del $2009\left(1^{\circ}\right.$ trim $\left.2009=100\right)$. La operación a realizar consistiría en el cociente entre el periodo final y el periodo base, y el producto de ese resultado por 100.

En lo concerniente a los ingresos totales hoteleros y extrahoteleros, el procedimiento planteado sería el mismo, con la única diferencia de la encuesta de la que se extraerían los datos (Encuesta de Alojamiento en Establecimientos Hoteleros y Encuesta de Alojamiento en Establecimientos Extrahoteleros).

Para el cálculo del indicador se ha llevado a cabo, en primer lugar, la recopilación de los datos relativos al gasto turístico total en destino para todas las islas en el periodo comprendido entre el $1^{\circ}$ trimestre de 2009 y el $1^{\circ}$ trimestre de 2013. Posteriormente, se calculó el cociente del dato para el periodo de referencia entre el dato del periodo base, obteniéndose así un índice para cada isla y trimestre. La misma operación fue llevada a cabo para el caso de los ingresos hoteleros y extrahoteleros. Una vez obtenidos todos los índices, pasaron a agruparse en uno solo los relativos a ingresos, mediante la suma del índice de ingresos hoteleros y el de ingresos en apartamentos correspondientes a cada isla y trimestre. Se dividió entre 2, por tratarse de dos índices resultantes, y se multiplicó por 100. Exactamente el mismo procedimiento se siguió para obtener un índice común a partir de los 3 indicadores.

Fue a la hora de establecer la metodología para el cálculo del índice desagregado cuando surgieron los problemas que hicieron replantear la decisión de si incluir o no este indicador en el presente estudio.

Una vez obtenidos los índices agregados, el siguiente paso consistiría en aportar esta misma información, pero de manera desagregada, por mercados emisores. Para ello se utilizarían los datos relativos al gasto en destino y los ingresos en establecimientos hoteleros y extrahoteleros para cada isla, desglosado por los 
siguientes países emisores: Alemania, España, Holanda, Países Nórdicos, Reino Unido y un último apartado en el que se agruparían el resto de países ("Otros países"). En el caso de España, surgió la duda de si incluir los datos referentes a los ingresos generados por los residentes canarios en otras islas, teniendo en cuenta que en la Encuesta sobre Gasto Turístico no se incluye el efectuado por los mismos.

Superado el paso anterior, se procedería a establecer el peso del gasto en los ingresos de cada mercado emisor dentro del total de cada isla para cada periodo. Para la obtención del índice desagregado, se multiplicaría la ponderación correspondiente a cada mercado emisor para cada isla y periodo por el índice obtenido inicialmente, en el paso anterior.'

En un intento de validar esta metodología, se realizaron una serie de pruebas utilizando datos reales extraídos de la web del ISTAC, con el fin de compararlos con los índices agregados y desagregados obtenidos. Al plantear el índice agregado, todo parecía apuntar a que el modelo era válido, pues los índices que se habían definido coincidían en buena medida con los datos reales; sin embargo, esto no era suficiente, y es que la suma de los índices desagregados resultantes de cada nacionalidad para un periodo debía dar el mismo resultado que el obtenido en el índice agregado. En un principio se detectó que en el caso de los ingresos, dichos resultados sí coincidían pero, en cambio, en el caso del gasto, no.

Tras analizar detalladamente los resultados, se llegó a la conclusión de que el peso de cada nacionalidad podía alterar el resultado global. Así, por ejemplo, se observó que el gasto total en Lanzarote en términos absolutos cayó entre el $1^{\circ}$ y el $2^{\circ}$ trimestre del 2012, y, sin embargo, el índice obtenido indicaba que había aumentado. La razón de ello se encontraba en el gasto de los británicos, que constituían el mercado con mayor peso y cuyo gasto, sobredimensionado, se había incrementado.

Planteado este problema, se comenzó un proceso de revisión exhaustiva de más publicaciones referentes a la metodología para la construcción de un indicador sintético, pero por no disponer de tiempo suficiente para su correcta definición, se ha acordado, junto con el ISTAC, que este trabajo de campo sirva como una base sobre la que seguir trabajando en un futuro a la hora de construir el índice sintético previamente definido.

\section{Descripción del sistema de indicadores propuesto}

Como se ha definido en apartados anteriores del presente documento, el sistema de indicadores propuesto se presenta como una herramienta muy útil ante la gran cantidad de información sobre coyuntura turística de la que disponen los gestores turísticos a la hora de tomar decisiones, en la medida en que ofrece, mediante un formato de sencilla visualización, solo los indicadores más relevantes para explicar la situación coyuntural de una isla o un municipio turístico, seleccionados bajo los criterios de nivel de uso y opinión de expertos del sector. 
La información contenida en el sistema de indicadores propuesto se puede contemplar a diferentes niveles territoriales:

- Autonómico, pudiendo acceder a un cuadro con los datos correspondientes a Canarias

- Insular, con la posibilidad de generar, de esta forma, 7 cuadros diferentes, coincidiendo con cada una de las islas: Lanzarote, Fuerteventura, Gran Canaria, Tenerife, La Gomera, La Palma y El Hierro

- Municipal, englobándose así los principales municipios turísticos más importantes del Archipiélago sobre los que el ISTAC publica datos: Arrecife, Teguise, Tías, Yaiza, Antigua, La Oliva, Pájara, Puerto del Rosario, Mogán, Las Palmas de Gran Canaria, San Bartolomé de Tirajana, Adeje, Arona, Puerto de La Cruz, Santa Cruz de Tenerife, Santiago del Teide, Breña Baja y Fuencaliente. En el caso de La Gomera y El Hierro solamente se ofrecerá el dato insular. En lo referente a este último nivel territorial, cabe señalar que la disponibilidad de los indicadores será más limitada que en los casos anteriores, por existir variables para las que el mínimo geográfico disponible es el insular

El usuario que acceda al sistema tendrá a su disposición un listado con los 15 indicadores anteriormente mencionados, dispuestos de forma vertical, en donde podrá consultar el último dato publicado en la web del ISTAC para Canarias, o para la isla o municipio seleccionado. Acompañando al nombre del indicador, encontraríamos, en la siguiente columna a la derecha, un botón identificado con una $\mathrm{i}$ de información mediante el que es posible acceder a breves notas metodológicas que faciliten la comprensión de los datos mostrados por parte del usuario. En este apartado, además, el usuario podrá conocer la operación estadística y la fuente de la que fueron extraídos los datos del indicador en cuestión, acompañado de un enlace a la web del ISTAC, y la fecha de publicación del siguiente dato. Anexo a éste, se localiza un botón llamado Evol. (Evolución), donde mediante un click del usuario, el sistema generará un gráfico con la evolución del indicador en cuestión. A la derecha de este botón, encontramos otra columna, a la derecha, denominada periodo, en la que se hará referencia al mes o trimestre al que corresponde el último dato publicado. En el resto de columnas, el usuario se encontrará con la información referente al indicador correspondiente en forma de:

- Dato. Al hablar de dato, nos referimos a una "serie que representa en cada observación el valor adquirido por una determinada variable en un determinado período de tiempo, sin relación alguna con otros períodos de tiempo previos o con otras variables". 
- Dato acumulado. "Serie cuyas observaciones recogen, de forma acumulada, los valores adquiridos por la variable a lo largo de un determinado período de tiempo y todos los anteriores al menos dentro de un marco temporal más amplio".

- Tasa de variación interanual. "Se dice que una serie está medida en tasas de crecimiento interanual cuando cada valor expresa el incremento del valor de la serie respecto al mismo periodo del año anterior." Por ejemplo: octubre del 2010 con respecto a octubre del 2009, o tercer trimestre del 2007 sobre tercer trimestre de 2006. Teniendo en cuenta que los indicadores seleccionados serán publicados con frecuencia mensual o trimestral (salvo en el caso de los datos sobre los turistas llegados a El Hierro, que serán semestrales), las fórmulas a tener en cuenta para el cálculo de las tasas de variación interanuales son las siguientes:

Para series mensuales:

Para series trimestrales:

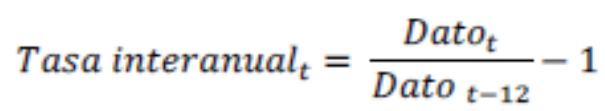

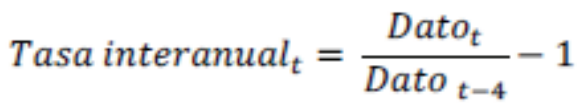

Tal y como apunta el profesor de la Universidad Autónoma de Madrid, Ramón Mahía (2001), las tasas interanuales cuentan con una gran ventaja con respecto a otras tasas de crecimiento, como las interperiodo, y es que "son insensibles al problema de la estacionalidad, lo que resulta, evidentemente, muy recomendable cuando se desea conocer un movimiento tendencial".

- Tasa de variación interanual acumulada. "La tasa acumulada indica, para un mes o trimestre dado, el crecimiento acumulado a lo largo de los meses o trimestres transcurridos hasta la fecha desde inicio del año:

a) Si la variable es de stock o es un índice, la tasa acumulada puede calcularse de forma sencilla comparando el período actual con el valor de cierre del pasado año.

b) Si la variable es de flujo puede compararse el promedio o la suma simple de la variable en los meses transcurridos del año en curso, con el promedio o la suma de la variable durante los mismos meses del año anterior".

Además de mostrar el dato del periodo presente, el dato acumulado, la variación interanual y la variación interanual acumulada, el sistema facilitará la visualización 
de la información mediante colores: verde en los casos en los que la tasa de variación sea positiva, amarillo cuando ésta se mantenga estable (es decir, cuando sea del $0 \%$ ), y rojo en los supuestos en los que la variación sea negativa. Asimismo, para no ceñirse únicamente a ofrecer simples cifras, el sistema proporcionará información adicional en el caso de algunos indicadores. Así, por ejemplo, en el supuesto de las pernoctaciones hoteleras y extrahoteleras, el usuario tendrá a su disposición un desglose de la información en función de la procedencia de los viajeros: residentes en el extranjero y residentes en España (incluyendo Canarias). En el caso del gasto medio por turista y día, se ofrece el dato del realizado por los turistas procedentes de Alemania, España (excluyendo Canarias) y Reino Unido, por considerarlos los principales mercados emisores, a fin de realizar comparaciones entre ellos y con respecto a la media de todos los países. Los mismos mercados emisores han sido tenidos en cuenta en el supuesto de las llegadas de turistas a la isla que han elegido como destino principal, indicador para el que se presenta el peso de cada uno de los mercados emisores en el total de las llegadas (al igual que en el gasto, en el dato de las llegadas procedentes de España no se incluye Canarias). Igualmente, el usuario tendrá la posibilidad de seleccionar, además de la visualización del dato y la representación gráfica de la evolución del indicador seleccionado desde el año 2009, la consulta de series históricas correspondientes a periodos anteriores. A estas características habría que añadir otra ventaja adicional y es que, mediante un código html, el cuadro generado podría ser incrustado como widget en otra página web para la que esta herramienta pueda ser de interés, como es el caso de las vinculadas a asociaciones empresariales, patronatos municipales de turismo, grupos de investigación, docencia virtual, etc.

\section{CONCLUSIONES}

En un contexto como el actual donde la información en materia de coyuntura turística, lejos de representar una carencia, ha llegado a suponer un exceso, ser capaces de seleccionar solo aquellos datos realmente relevantes se vuelve, sin duda, una ardua tarea. Más se acentúa esta dificultad si dicha información va a servir de respaldo para una adecuada toma de decisiones por parte de una empresa o una Administración Pública vinculada al sector turístico, puesto que una decisión errónea puede conducir al fracaso absoluto. En el caso de Canarias, el Instituto Canario de Estadística (ISTAC) ha venido desarrollando una labor formidable en lo que a recapitulación de información sobre coyuntura turística se refiere, realizando publicaciones de importante frecuencia y que en muchos casos alcanzan un nivel territorial altamente desagregado, entendiéndose que dentro de una misma isla existen realidades muy heterogéneas $\mathrm{y}$, por tanto, lo más adecuado es tratarlas por separado. Sin embargo, desde el punto de vista del usuario, cuando éste visita por primera vez ésta u otras páginas web que contengan este tipo de información, lo 
cierto es que la mayor parte de las veces no se es capaz de acceder directamente a los datos que se buscan, sino que se tiende a vagar por otros apartados llegando incluso a abandonarse esta labor de búsqueda. Y es que, si no disponer de información es un problema, contar con un exceso de ésta también lo es, ya que pone a prueba nuestra capacidad para discernir y en un entorno en el que exista ruido, éste no es un cometido sencillo. A esto hay que sumar también la incomodidad que supone tener que visitar diferentes webs o, dentro de una misma, distintos apartados, para acceder a la información, con el riesgo de, por ejemplo, no recordar dónde se había visto aquella información tan interesante que no se guardó.

Dar solución a estos problemas es la razón de ser del presente proyecto. Sin embargo, como ya se ha apuntado anteriormente, la idea de llevar a cabo esta iniciativa no es nueva, sino que es fruto de la preocupación compartida por la Cátedra de Turismo de la Universidad de La Laguna y del ISTAC, por lo que, más allá de un trabajo de interés meramente académico, se trata de una especie de proyecto piloto que se llevará a la práctica por parte de estos dos organismos.

La propuesta realizada se basa en la construcción de un sistema de indicadores de coyuntura turística en el que se hayan seleccionado previamente una serie de indicadores, 15 en este caso, que permitan intuir de manera rápida y sencilla si el sector turístico de una isla o un municipio turístico marcha favorablemente o no. Para ello, se han tenido que seguir una serie de fases, que se pueden resumir en tres:

1- Recapitulación de todos los indicadores relacionados con coyuntura turística publicados a nivel autonómico, nacional e internacional, por medio de la consulta a fuentes como: Instituto Canario de Estadística, Cabildo de Tenerife, Instituto Nacional de Estadística, Instituto de Estudios Turísticos, Exceltur, Eurostat y Organización Mundial del Turismo. Como resultado de este proceso se obtuvieron más de 120 indicadores sobre coyuntura que engloban diferentes dimensiones de análisis.

2- Selección de los indicadores más relevantes de los que fuera posible obtener información para Canarias, resultando un total de 15. Cabe aclarar que la elección de dichos indicadores no ha sido aleatoria, sino que se ha realizado en base a criterios objetivos, como el nivel de uso (mediante el número de veces que el usuario hace click en cada uno de ellos), y otros más subjetivos, como la opinión de expertos del sector público y privado.

3- Planteamiento inicial de un indicador sintético a partir del gasto realizado en destino por los turistas, los ingresos totales hoteleros y los extrahoteleros, sobre el que se solo se ha sentado una base sobre la que seguir trabajando en futuros proyectos por encontrarnos con problemas de tipo metodológico.

El sistema al que se hace referencia, además de permitir una visualización rápida y sencilla de los indicadores, cuenta con una serie de ventajas básicas con respecto a otras publicaciones similares que se realizan en Canarias, como es el caso de las correspondientes a las Cámaras de Comercio: 
- Contará con un sistema de actualización instantánea y permanente, de forma que siempre aparezca recogido el último dato publicado en la página web del ISTAC

- El periodo de referencia para la publicación de los indicadores será el mensual, salvo que éstos solo puedan obtenerse con carácter trimestral. Asimismo, se podrá acceder a un mínimo geográfico equivalente al municipal, salvo en los indicadores en los que esto no sea posible

- Podrá consultarse, no solo el último dato disponible, sino también otros publicados con anterioridad en un apartado denominado Series históricas, dispuestos tanto en forma de tabla como de gráfico

- El sistema detectará el signo de las tasas de variación y asignará el color rojo, en el caso de los porcentajes negativos, verde, en los positivos, y ámbar en los indicadores en los que no se han producido variaciones, permitiendo así una interpretación de la información más visual e intuitiva

- Existirá la posibilidad de construir un widget $\mathrm{html}$ en la medida en que se permitirá incrustar el cuadro generado en páginas web de asociaciones empresariales, ayuntamientos, empresas, universidades, etc

- Se tratará de un sistema personalizable, en donde el usuario tendrá la oportunidad de incluir algunos indicadores no recogidos en la selección predeterminada o excluir aquellos que no son de su interés

A lo largo de estas páginas, el presente proyecto ha venido a desarrollar solo el inicio de uno de los tantos caminos que pueden seguirse en este sentido. Por un lado, se considera que se podría avanzar un paso más si los usuarios, de forma individual, pudieran establecer una serie de objetivos cuantificables para cada uno de los indicadores y el sistema, una vez publicado el dato correspondiente, detectara si dichos objetivos se han alcanzado o no, asignando en cada caso un símbolo. Igualmente, ha de seguir trabajándose en aspectos relacionados con la metodología, para definir correctamente el indicador sintético que no ha sido posible desarrollar en este proyecto. Otro punto en el que habrá que trabajar será en lo referente al mínimo geográfico al que será posible acceder. En este punto hay que mencionar el trabajo que está desarrollando la Cátedra de Turismo de la ULL de forma conjunta con el ISTAC sobre microdestinos de Canarias, sentándose así las bases sobre la que construir en un futuro este mismo sistema a este nivel geográfico aportando datos para diferentes zonas de un mismo municipio. También podría barajarse en el futuro la posibilidad de aportar los datos sobre gasto a precios constantes, en lugar de a precios corrientes, para que éste no se vea afectado por la inflación. Por último, habría que trabajar en un sistema de evaluación del funcionamiento del sistema como forma de obtener feedback. La aparición de preguntas del tipo “¿Le han sido útiles estos indicadores?” y “¿Qué indicadores no publicados añadiría?" podría ser una forma de hacerlo. 


\section{BIBLIOGRAFÍA}

AENOR, (2003). Norma UNE 66175:2003. Guía para la implantación de sistemas indicadores. AENOR Ediciones.

Cámara de Comercio de Santa Cruz de Tenerife (2013). Informe sectorial $1^{\circ}$ trimestre 2013.

CASTRO NÚÑEZ, R.B. y LÓPEZ GARCÍA, A.M. (2004). Valoración de la actividad económica regional de España a través de indicadores sintéticos. Instituto L.R.Klein - Centro Stone, Madrid.

Dateco Consultores S.L para el Instituto Canario de Estadística (1998). Indicador de actividad turística de Canarias. Descripción y mantenimiento de ficheros.

DEL SUR MORA, A. y BARRIGA RINCÓN, L. (2000). Indicadores sintéticos de actividad. Instituto L.R. Klein, UAM. Madrid.

ESPASA, A. y CANCELO, J.R. (1993). Métodos Cuantitativos para el análisis de la coyuntura económica. En: Estudios de economía aplicada, Vol. 22, № 3, 2004 (Ejemplar dedicado a: Economía espacial), pp. 631-656. Alianza Editorial, Madrid.

Eurostat (2005). Código de buenas prácticas de las estadísticas europeas.

Eurostat (2012). Socio-economic analyses of the regions. NUTS 3: small regions for specific diagnoses. Clasificación válida de enero de 2012 a diciembre de 2014.

Eurostat (varios años). Main tables y Database.

Exceltur (varios años). Indicadores Sintéticos del Turismo en España, en Anexos Metodológicos, Perspectivas Turísticas, pp 106- 107. Madrid.

Exceltur (varios años). ICTUR (Índice de Confianza Turístico).

Exceltur (varios años). ISTE (PIB turístico)

Exceltur y Gobierno de Canarias (2012). Estudio del impacto económico del turismo sobre la economía y el empleo de las Islas Canarias. Impactur Canarias 2011.

Instituto Canario de Estadística (2002). Indicador Sintético de la Actividad Turística de Canarias. ISTAC, Canarias.

Instituto Canario de Estadística (varios años). Encuesta de Alojamiento Turístico en Establecimientos Hoteleros y Encuesta de Alojamiento Turístico en Establecimientos Extrahoteleros, ISTAC, Canarias.

Instituto Canario de Estadística a partir de datos del Instituto Nacional de Estadística, (varios años). Encuesta Anual de Servicios, ISTAC, Canarias.

Instituto Canario de Estadística (varios años). Encuesta sobre Gasto Turístico, ISTAC, Canarias.

Instituto Canario de Estadística (varios años). Encuesta de Expectativas Hoteleras, ISTAC, Canarias.

Instituto Canario de Estadística (varios años). FRONTUR- Canarias. Estadística de Movimientos Turísticos en Fronteras de Canarias, ISTAC, Canarias. 
Instituto Canario de Estadística,(varios años). Estadística de Empleo Registrado, ISTAC, Canarias.

Instituto Canario de Estadística a partir de datos del Servicio Público de Empleo Estatal, (varios años). Estadística de Movimiento Laboral Registrado, ISTAC, Canarias.

Instituto Canario de Estadística (varios años) a partir de datos del Instituto Nacional de Estadística. Encuesta de Población Activa, ISTAC, Canarias.

Instituto Canario de Estadística (varios años) a partir de datos del Ministerio de Empleo y Seguridad Social. Estadística de Empresas Inscritas en la Seguridad Social.

Instituto de Estadística y Cartografía de Andalucía, IECA (2012). Indicadores de Actividad del Sector Servicios, Metodología base 2010.

Instituto de Estadística y Cartografía de Andalucía, IECA (2012). Indicadores Estadísticos de Andalucía

Instituto de Promoción Turística de Castilla- La Mancha y Universidad de Castilla- La Mancha (2008). Indicadores de Actividad Turística, Documento de Trabajo 4.

Instituto Nacional de Estadística (2008). Índice de Precios de Consumo (varios años), INE, Madrid.

Instituto Nacional de Estadística (2011a). Indicadores de actividad del sector servicios (IASS), base 2005. Nota metodológica. Madrid.

Instituto Nacional de Estadística (2011b). Indicadores de actividad del sector servicios (IASS), base 2010. Nota metodológica. Madrid.

Instituto Nacional de Estadística (varios años). Índice de precios hoteleros, Índice de precios de acampamentos turísticos, Índice de precios de apartamentos turísticos e Índice de precios de alojamiento de turismo rural. Madrid.

Linkedin, Destination Management Organisations. [Consulta: marzo de 2013].

LÓPEZ, M.T. y GENTILE, N. (2008). Sistema de indicadores económicos y sociales: la importancia del análisis integrado. In: Encuentro Nacional de la Red de Economías Regionales en el Marco del Plan Fénix, 9. Jornadas Nacionales de Investigadores de las Economías Regionales, 2, 18-19 septiembre 2008, Tandil. Argentina.

MAHÍA, R. (2001). Conceptos básicos de matemática económica para el manejo de series.

MAS RODRÍGUEZ, P. (2009). Indicadores sintéticos de la economía española. En: Revista de estadística y sociedad, pp. 14- 17.

MASOLLER, A. (2001). Un indicador sintético de actividad económica. En: Revista de Economía Aplicada, no 004- 2001.

Ministerio de Economía y Hacienda, Dirección General de Política Económica,

Subdirección General de Análisis Macroeconómico (2007). Revisión de la metodología para el cálculo de los indicadores sintéticos de la economía española. 
Organización Mundial del Turismo, (2008a). Cuenta Satélite del Turismo: Recomendaciones sobre el marco conceptual. Madrid/Nueva York, 2010.

Organización Mundial del Turismo, (2008b). Recomendaciones internacionales para estadísticas de turismo. Madrid/Nueva York, 2010.

Patronato de Turismo del Cabildo de Gran Canaria, (2013). Situación del Sector Turístico, cierre año 2012.

Región de Murcia, Consejería de Turismo y Ordenación del Territorio, Secretaría General, Unidad de Estudios y Estadística, (2002- 2011). Sistema de Indicadores Turísticos de la Región de Murcia.

RODRÍGUEZ APONTE, D.Y. (2007). Algunos conceptos básicos sobre los indicadores.

RODRÍGUEZ- SALMONES, N. (1998). Los indicadores estadísticos de la economía del turismo en España. En: Revista valenciana d'estudis autonòmics, número 25, cuarto trimestre de 1998, pp. 45- 54.

SUR, A. (2000). Elaboración de un Indicador Sintético para la Comunidad de Madrid.

Instituto L.R. Klein. Jornadas XXVIII sobre Economía de la Ciudad de Madrid. Reunión Hispalink. Oviedo.

Turismo de Tenerife, Cabildo de Tenerife, a través de Autoridad Portuaria de S/C de Tenerife (varios años). Pasajeros de cruceros.

Turismo de Tenerife, Policía Turística del Cabildo de Tenerife (varios años). Plazas alojativas autorizadas (total), Plazas hoteleras autorizadas, Plazas autorizadas en apartamentos, Plazas autorizadas en hoteles rurales y Plazas autorizadas en casas rurales.

Unión Europea. Reglamento N ${ }^{\circ}$ 692/2011 del Parlamento Europeo y del Consejo de 6 de julio de 2011, relativo a las estadísticas europeas sobre el turismo y por el que se deroga la Directiva 95/57/CE del Consejo. 